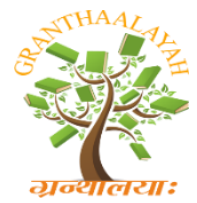

\author{
INTERNATIONAL JOURNAL OF RESEARCH \\ GRANTHAALAYAH \\ A knowledge Repository
}

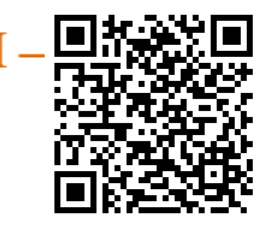

Science

\title{
EXPERIMENTAL INVESTIGATION ON THE ROLE OF THERMO- ACOUSTICS ON SOOT FORMATION
}

\author{
Rahul Ravi Ravichandran ${ }^{\text {, }}$, Sambit Supriya Dash ${ }^{2}$, Vinayak Malhotra *3 \\ $1,2,{ }^{* 3}$ Department of Aerospace Engineering, SRM Institute of Science and Technology, India
}

\begin{abstract}
Combustion is a complex phenomenon that involves the interaction of multiple phenomena, the cumulative effect of which give rise to the common flame that comprises wide range of practical, functional to propulsion applications. An interesting aspect that needs attention is the effect of the various surrounding environment phenomenon on its behavior and properties of premixed flames. It is noteworthy that soot formation is the main cause of pollution and a major cause of inefficiency of current propulsion systems. These phenomena root variety of energy interactions leading to energy transformations which in turn affect the flame behavior. Present work focuses on experimental investigations and implications of the externally induced acoustics on partially premixed flames. The subject is extensively studied as thermo-acoustics, and the current work emphases on studying its role on soot formation on premixed flames. Experimentation was carried out on a scaled setup comprising of a butane cylinder fitted with a nozzle, that houses 3 arrays consisting of 4 holes each placed equidistant to each other for entraining air. The resulting flame is impinged with acoustics from two independent and similar sources placed equidistant from the center of the nozzle. The entire process is systematically video graphed using a 60-fps regular $\mathrm{CCD}$ and analyzed for variation in flame heights and flickering frequencies. The observations involve fixed fuel mass flow rate with varying configuration of entrainment holes and acoustic frequency under normal ambient atmospheric conditions. The work establishes significant outcomes on the effect of acoustics on soot formation. The results are likely to encourage better understanding with applicability to commercial and domestic utilizations for energy generation for enhanced effectiveness and reduced hazards
\end{abstract}

Keywords: Premixed Flames; Thermo-Acoustics; Entrainment; Propulsion System; Efficiency.

Cite This Article: Rahul Ravi Ravichandran, Sambit Supriya Dash, and Vinayak Malhotra. (2018). "EXPERIMENTAL INVESTIGATION ON THE ROLE OF THERMO-ACOUSTICS ON SOOT FORMATION." International Journal of Research - Granthaalayah, 6(6), 461-471. https://doi.org/10.29121/granthaalayah.v6.i6.2018.1391.

\section{Introduction}

Combustion deals with exothermic chemical reaction by using fuel and oxidizer associated with a reaction zone. Fuel and oxidizer mix in required proportions and combustion occurs through an 
intricate sequence of reaction steps. Combustion is critically important and the resultant energy interactions and governing phenomenon sources close to $80 \%$ of the world energy requirements in form of like propulsion, heating, electricity production. This branch of physics is broadly classified into smoldering and flaming. The classification is based on the manifestation of flames resulting from highly exothermic reactions yielding high flame temperatures depending on the fuel-oxidizer ratio.

Different types of flames can result from the way in which the fuel and oxidant are mixed. Combustion can occur in premixed or diffusion modes. Diffusion flame represents diffusioncontrolled combustion with diffusion rate greater than the reaction rate. In diffusion flame, fuel and oxidizer concentration vanishes at flame front and the flame region is very thin. In premixed flames, fuel such as natural gas, commercial and industrial liquid fuels, usually termed fuel oils and air mixture kept in an open tube is lighted by a spark and the propagation of the flame is observed at a certain velocity. In comparison to the premixed flames (short and blue), diffusion flames are yellow, longer, luminosity, sooty and with higher stability range, and less flame temperature. When the fuel flow rate is relatively low, the incoming gaseous flow of fuel and air is laminar, as is the flame however; high fuel flows may lead them to being turbulent. Thus, flames are generally identified as to be laminar premixed, laminar diffusion, turbulent premixed or turbulent diffusion. In addition, they can also be categorized into stationary or propagating flames. Partially premixed flames contain a rich premixed fuel-air mixture in a stream, and, for complete combustion to occur, they require the transport of oxidizer from an appropriately oxidizer rich mixture that is present in another stream. The stationary flames are widely used in domestic or industrial burners, the propagating flames being involved in explosions. An important aspect of the combustion research is investigation of the behavior of flames in response to numerous parametric variations and external influences. The premixed and diffusion flames had been extensively studied and explored owing to the strong physical presence and applications. Combustion systems are one of the most important and complex highlighting an interesting aspect of the presence of partially premixed flames as a coupled resentment between premixed flame and diffusion flame. Flames in most practical applications cannot be described as purely premixed or non-premixed. Understanding the behavior of flame in response to multiple air-entrainments to fuel on its path to produce flame is important because of its wide range applicability i.e. ring pool fire, perforated liner in combustors, furnace burner rims, Bunsen burners, perforated exhausts and culinary flame torches. This study could be very effective for fire safety purpose of a multiplewindowed tall infrastructure. The parametric study of flaming combustion characteristics for ring pool fires and perforated liners has been done by researchers for decades. Scientists have stratified, classified and quantified the traits of flame in influence of amount of passive air-entrainment, forced air-entrainment, variation of composition of fuel and oxidizer and induced instabilities. One important aspect of the flaming combustion yet to be comprehensively resolved is the understanding of soot formation/oxidation. It is reported that a strong correlation between the deaths and particulates, realized repeatedly in many different locations \& situations exists and soot kills. With combustion, soot presents issues like much shorter life span, health impacts significantly and slows economic growth. At present, existing soot oxidation models are extremely simplistic. Models include irreversible reactions, but a in-depth solution is yet to be obtained, so something is fundamentally wrong.

Sohrab and Law [1] studied characteristics of polyhedral flames of propane and butane in influence of burner rim aerodynamics by illuminating the effects of atmospheric entrainment, inner wall 
boundary layer, and conductive heat loss to the burner. They found burner tips are unable to support polyhedral structure and rise in surrounding atmospheric Nitrogen enhances the propensity of polyhedral structure. The rotational speeds based linear velocities at times more than laminar propagation velocity corresponding to the same mixture. Wadia [2] gave a brief review on advanced liner cooling techniques focusing more on laminated porous wall cooling, angled-multihole (effusion) cooling and composite metal matrix liner by defining the concept of heat transfer considering material and fabrication problems associated with it. Jing and Sun [3] worked on the perforated liner in presence of a bias flow. They found that it gives significant rise in absorption coefficient and effective absorption bandwidth. The plate thickness also has a major significance on acoustic reactance and thus on resonating frequency. Eldredge and Dowling [4] investigated on effectiveness of cylindrical perforated liner with mean bias flow in its adsorption of planar acoustic waves in a duct. Each aperture was subjected to a harmonic pressure difference resulting vortex shedding from the rim. When the system included in a duct whose termination allows, most acoustic energy to reflect upstream for further interaction with the liner, can absorb as much as $83 \%$ of incident energy at certain frequencies, and prevent $100 \%$ of the outgoing energy from reflecting to the source. Lei, et. al., [5] worked for controlling combustion instability by the effects of perforated liners with bias flow and by analytically and experimentally found that perforated liners can largely suppress the combustion instabilities. Heuwinkel, et. al., [6] studied liner configuration under bias and grazing flow condition was performed in three different test facilities and with three different measurement techniques. Results show the influence of the flow dominates the damping behavior of the circular module and plane configuration shows an additional resonance effect. Though, the behavior outside of the resonance range is like the circular configuration. Rodrigues and Fernades [7] analyzed methane and propane flame stabilization on matrix-hole plate burner optimizing effects of hole diameter, distance between holes and number of holes of the flow distribution plate as function of the relative velocity gradient. The work showed that flames are less stable as large is the distance between holes on the distribution plate and that the flame stability is almost insensible to the other number of holes of the plate and the hole diameter and finally characterized the reacting propane airflow around burner plate. Lei, et. al., [8] investigated the detailed behaviors of the temperature, velocity (in axial and tangential directions) and air entrainment in fixed-frame type fire whirl plume. The radial temperature follows the decaying exponential function and the power exponent ' $n$ ' decreases from 2 to 1 with height in the intermittent flame and plume. In continuous flame, drop in increment rate of centerline axial velocity was observed comparative to buoyant flame.

Wang, et. al., [9] studied, the flame height and air entrainment coefficient of double buoyancycontrolled jet fires having two identical rectangular nozzles with same mass flow rate but varying distances. They found the flame height increases with the heat release rate, and it decreases with the distances between two nozzles if the distance is small. The flame height remains unchanged when the distance is large enough. $\mathrm{Hu}$, et. al., [10] experimentally investigated the evolution of flame height produced by line-source buoyant turbulent non-premixed jets with air entrainment constraint by two parallel side walls at various separation distances. Resulting in little flame height change with side walls separation distance when the longer side of the line-source nozzle is perpendicular to the side walls. Flame height was noted to decrease with increase in side walls separation distance. In recently, Tao, et. al., [11] experimentally inspected the effects of various diameters for ring pool fire resulting flame height of ethanol changes slightly and for n-heptane, it increases with equivalent diameter. They found a classic correlation for $n$-heptane and ethanol for plotting the evolution of flame height. The fuel flow rate and the extent of axial shear for fuel flow 
rates are major functions of soot emissions. Soot suppression increased along with higher external shear for all the fuel flow rates. Deepika, et. al., [12] observed that the soot suppression and flame area enhancement are contemporary following non-linearity, which reflects the flame to soot area variation across the magnitude of external shear for different fuel flow rates. Present work primarily focuses on simplifying and understanding the behavior of flames with varying location and for various sets of locations of air-entrainment zones of equal enclosure area. The work is motivated by the idea of enhanced understanding of flames as a function of various air-entrainment enclosure locations and simultaneous acoustics. The specific objectives of the work are:

- To study the effect of thermo-acoustics on soot formation.

- To investigate the coupled effect of varying entrainment area and acoustics on flame height and flickering frequency.

\section{Experimental Setup and Solution Methodology}

To address the objectives, an experimental setup (fig. 1) was upraised comprising of components, (a) Height reference chart, where each square box is $5 \mathrm{~mm}$, (2) Laptop connected to speakers with $\mathrm{NCH}$ tone generator software (fig. 6) for sound generation of varied frequencies, (3) Two acoustic sources identical in dimensions, power input and output characteristics, (4) Cylinder holder apparatus, (e) Butane cylinder. The cylinder and the sound sources are calibrated in vertical and horizontal positions for same level using the lines as seen from top view (please refer to fig. 2). The acoustic sources (speakers) are placed $20 \mathrm{~cm}$ from the center of nozzle on both sides are totally $40 \mathrm{~cm}$ from each other. The speakers were checked and corrected for perpendicularity and symmetry in position about all three directions of space. Selected nozzle contains 3 sets of 4 holes each (diameter $4.87 \mathrm{~mm}$ each) for required air entrainment. The array of holes positioned at the start of the nozzle are named E1, the one in middle of the nozzle are named as E2 and the array of holes positioned at the end of the nozzle are named as E3 (as shown in figure 3a). The experiments were carried out in normal gravity $\left(9.81 \mathrm{~m} / \mathrm{s}^{2}\right)$ and standard atmospheric conditions where temperature was maintained constant at $29^{\circ} \mathrm{C}$ and $21 \%$ oxygen concentration environment. The fuel mass flow rate was maintained constant by measuring the velocity of fuel flow from the orifice at the start of the nozzle as depicted in figure.3b. The fuel mass flow rate was maintained at $\mathbf{1 . 2 1 7}$ mg per second. Different configurations of entrainment holes were achieved by closing 3 holes completely and half of the fourth hole in a single row by using paper and adhesive tape. A case where all the holes are open is achieved as shown in figure.3(d). The ignition through external source is done at the end of the nozzle outlet in all cases to maintain uniformity as shown in figure.3e. Using $\mathrm{NCH}$ tone generator software, the sound sources produced sounds of frequencies $100 \mathrm{~Hz}, 500 \mathrm{~Hz}, 1000 \mathrm{~Hz}, 2500 \mathrm{~Hz}, 5000 \mathrm{~Hz}, 7500 \mathrm{~Hz}$ and $10000 \mathrm{~Hz}$ (refer fig. 4).

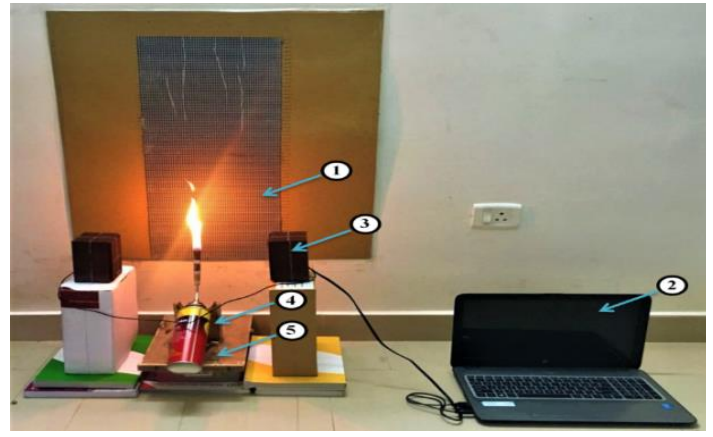

Figure 1: Complete experimental setup

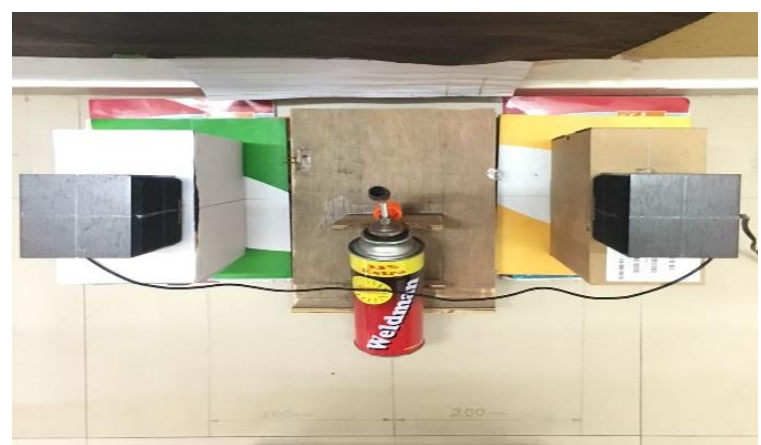

Figure 2: Top view of experimental setup 

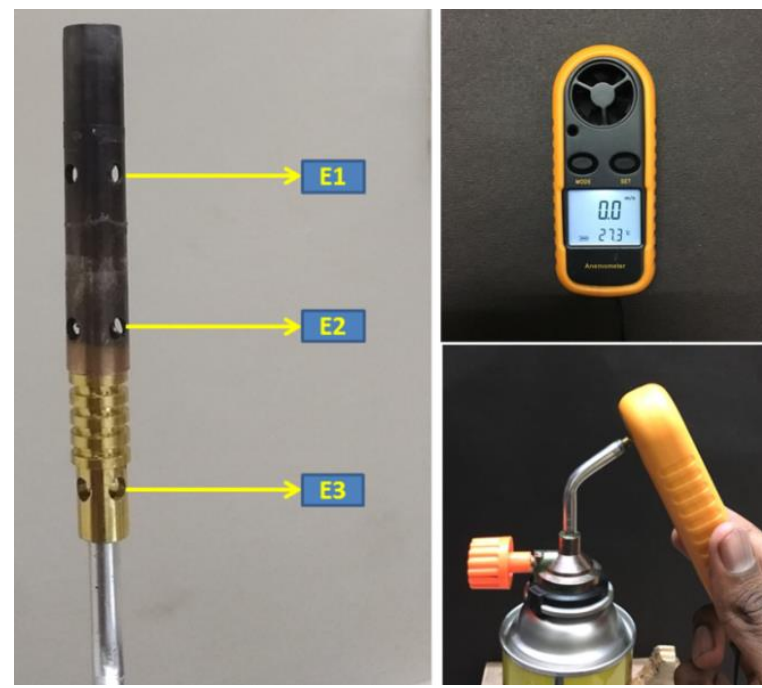

Figure 3: Essential components of experimental setup (left to right) viz., nomenclature of array of holes, Anemometer and flow measurement, All open case, Removable sub-parts of nozzle

After every case the flame was blown out and the apparatus could cool down for 10 minutes, so that the wall temperature of nozzle and atmospheric conditions remain constant throughout the experimentation duration. This process was repeated in all configurations of entrainment holes and each frequency of sound source. The orientation of the nozzle was always maintained perpendicular and equidistant from sound sources. The center point of the sound source was aligned to be in line with the entry point of nozzle in all cases. For each of the five entrainment hole configurations (only E1 open, only E2 open, only E3 open, all open and all closed) cases, firstly the fuel was calibrated to required level and ignited at the end of the nozzle and sound sources were switched on for the required frequency and allowed to impinge sound on the flame for 60 seconds, meanwhile a 60 -fps camera was set in position to video graph the entire process (refer fig.5). Later the video graph was shredded to obtain images at 12, 24, 36, 48 and 60 seconds time interval and the corresponding images were analyzed.

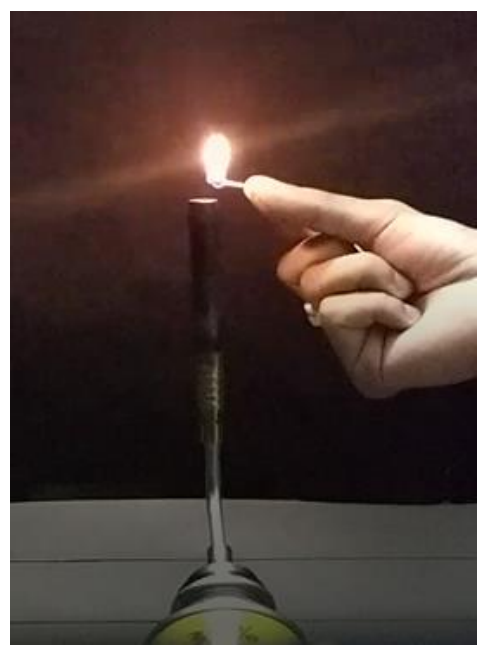

Figure 4: Ignition from top

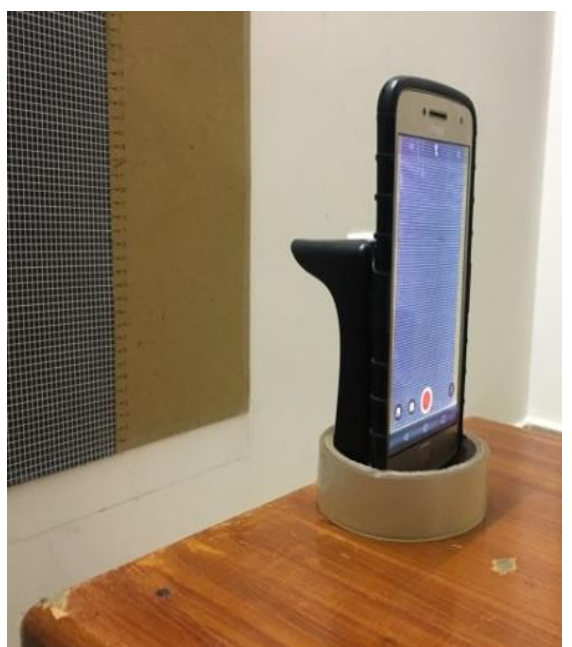

Figure 5: Camera stand and holder 


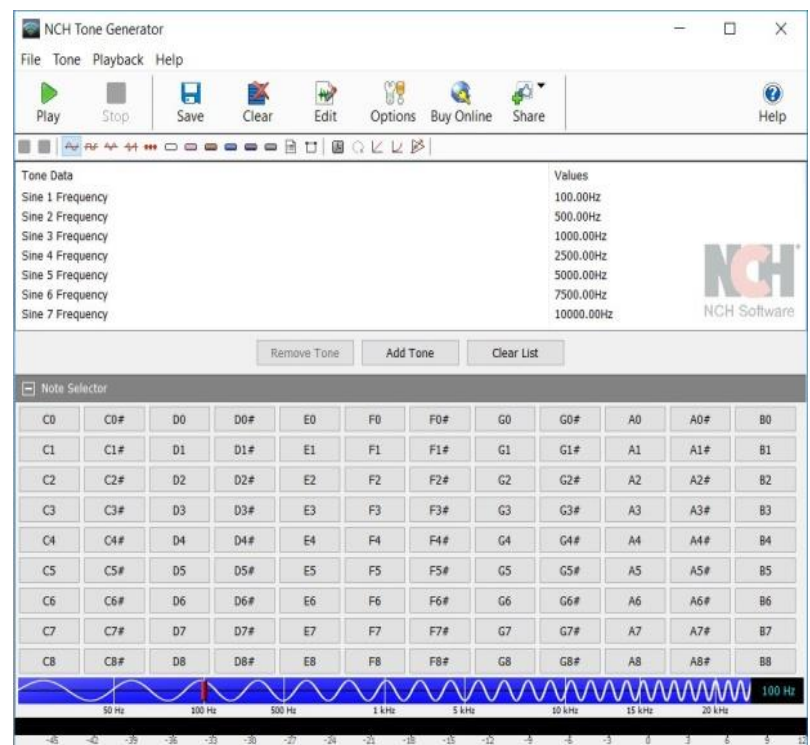

Figure 6: Screenshot of NCH tone generator

After every case the flame was blown out and the apparatus could cool down for 10 minutes, so that the wall temperature of nozzle and atmospheric conditions remain constant throughout the experimentation duration. This process was repeated in all configurations of entrainment holes and each frequency of sound source.

\section{Results and Discussions}

Stepwise experimentation was carried out to analyze the soot formation and alteration owing to the competing phenomenon of varying air entrainment and acoustics. First, a base case was established with only E1 kept open (viz., 3 holes of E1 completely closed and one hole is half closed) and no acoustic interaction with environment. The base case is primarily utilized for comparison owing to parametric variations.

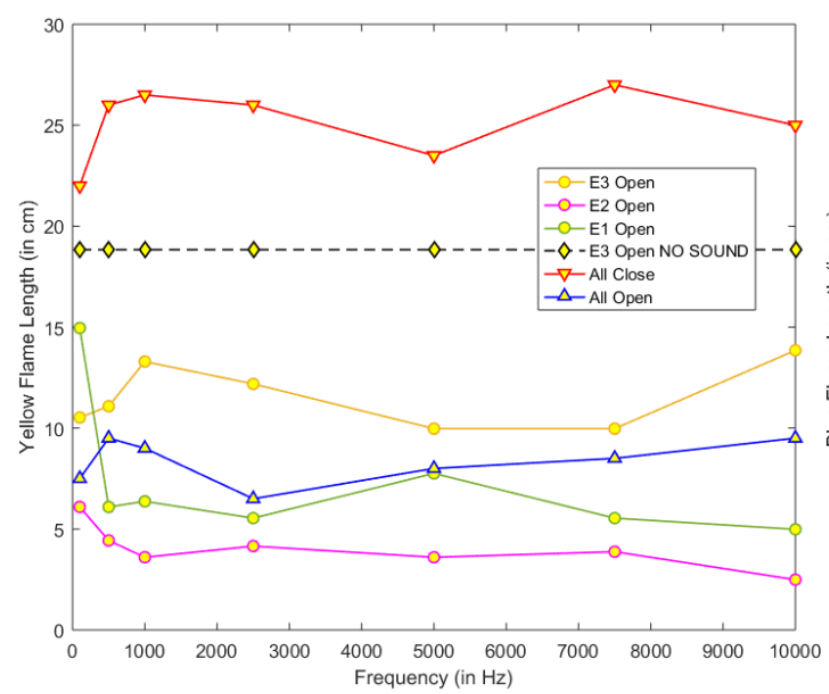

Figure 7: Variation of Yellow Flame Length. with frequency

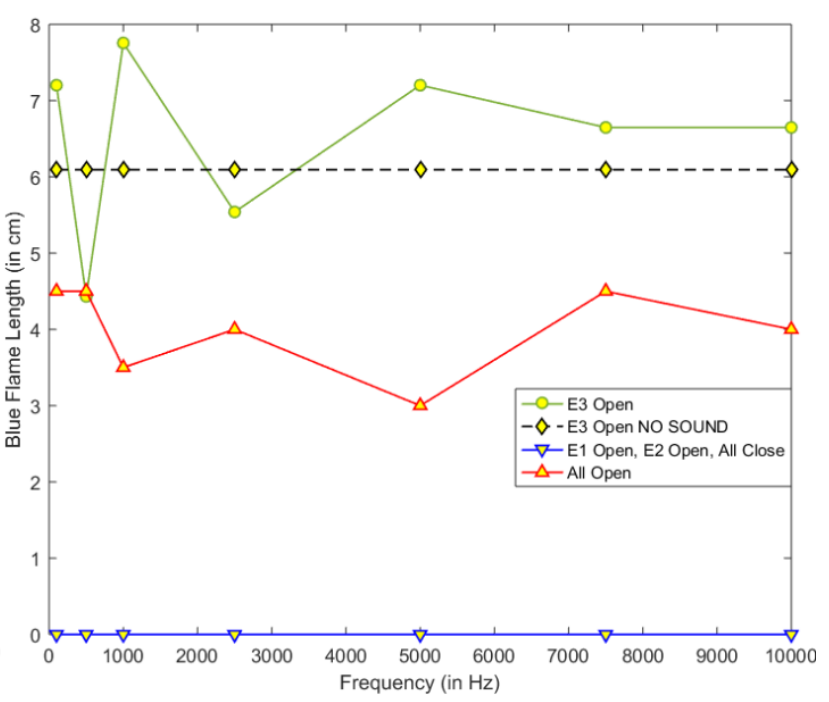

Figure 8: Variation of Blue Flame Length with frequency 
It is important to note that, the base case is represented by a straight line representing constant flame height. The provides a good validation to the experimentation because, E3 open signify, proper mixing of entrained air giving rise to a premixed flame. Further, with no acoustic energy interaction, it is expected to perceive a steady flame highlighting normalcy of the environment. To simplify the soot analysis, Gross flame length (GFL), Blue Flame Length (BFL) and Yellow Flame Length (YFL), were defined and their variation for different cases were tracked. Figure 7 highlights the variation in the YFL for diverse combinations of air-entrainment coupled to the varying acoustics impinged on the base flame. The sooty flame was noted to emanate from the trailing edge of premixed blue color flame with $3 \frac{1}{2}$ out of 4 holes of E3 set covered. Looking at the plot one can note that, for most of the cases the YFL stands below the base length. However, for all open case, escalated increase in YFL was observed. Selected cases of viz., All open, E1 open, E2 open, E3 open marks reduced YFL in a non-monotonic trend than the base case. Careful observations details that, the case with E3 open yields minimum drop from the base case followed by all open, E1 open and E2 open. The variation in YFL reveals effective inter energy conversion to understand the coupled effect of acoustics and varying air-entrainment. Figure 8 shows corresponding BFL variation with acoustics. E3 open results in distributed variation across the base case. Minimum BFL was accounted below $1000 \mathrm{~Hz}$ whereas, the maximum was accounted at $1000 \mathrm{~Hz}$. The variation of the flame lengths for the cases of E1, E2 open and all closed case shows that the blue flame height is zero in these cases. It is interesting to note that at higher acoustic frequencies, the rate of BFL change reduces. The interchanging BFL and YFL represents a certain interrelation with soot formation and acoustics.

Figure 9 shows the pictorial representation (taken at equal intervals of 12 seconds) of the experimentation carried out for cases mentioned above. Looking at the images, it can be noted that, as the entrained air mixes at a later stage, flame transits to diffusion as the measure of effective mixing reduces. Thus, the soot formation dominates giving rise to the yellow flame with almost negligible blue flame height. However, at lower acoustic frequencies, the flame length varies the most but beyond limit acoustic variation doesn't causes significant effect on the flame length. Similarly, observation for the case where all the holes are closed shows a resultant flickering flame. However, a structured blue flame as compared to the length of the yellow flame was configured. The unremitting variation in air-entrainment aids in the flaming transition, and with mutual acoustic energy interactions leading to the incomplete combustion with consequential yellow part of flame representing the soot or the partially burnt carbon particles and radicals. Further analysis of the images details the flickering behavior of flames resonating with soot formation. The series of snapshots (fig. 9) depict flame structure and shape changes with acoustic variation. It was observed that the flame in all the cases is shorter compared to the diffusion flame and most of the flame is blue with the yellow tip. However, the flame is unsteady and is flickering. 


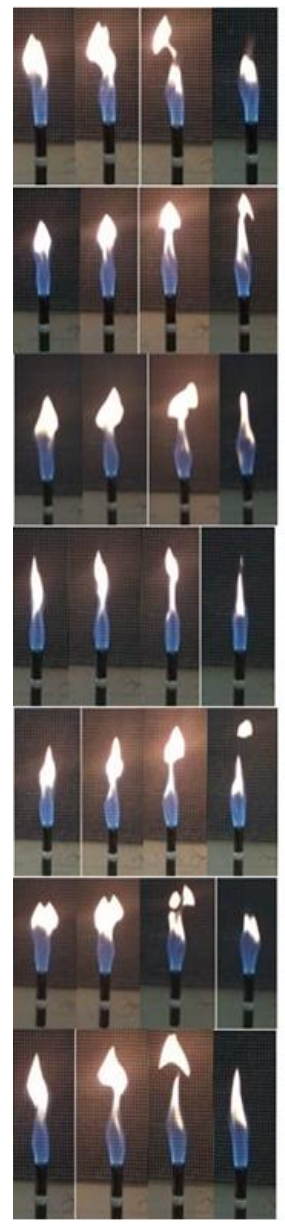

(b)

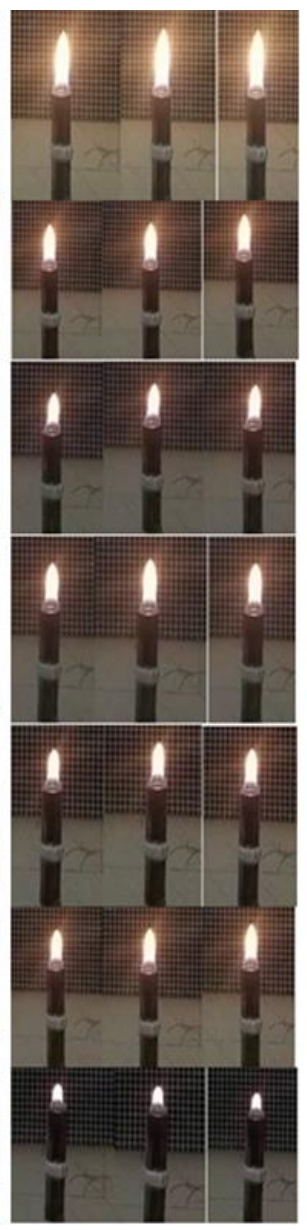

(c)

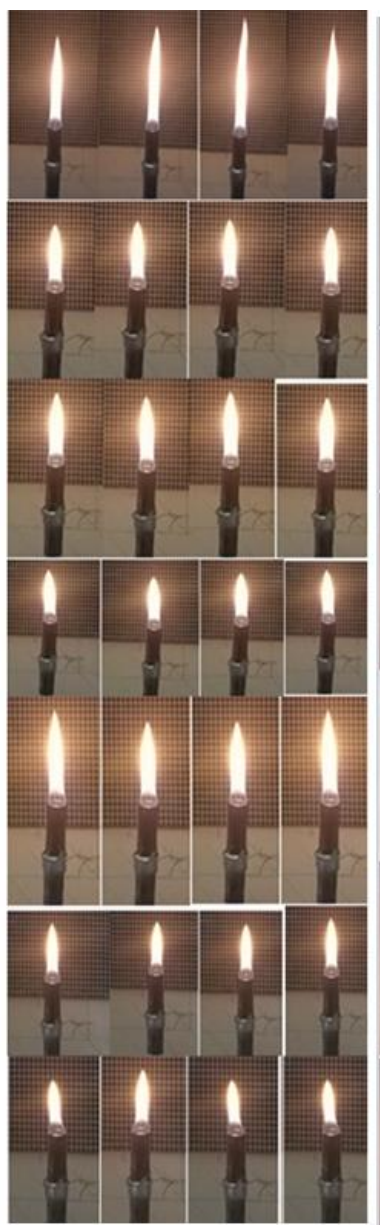

(d)

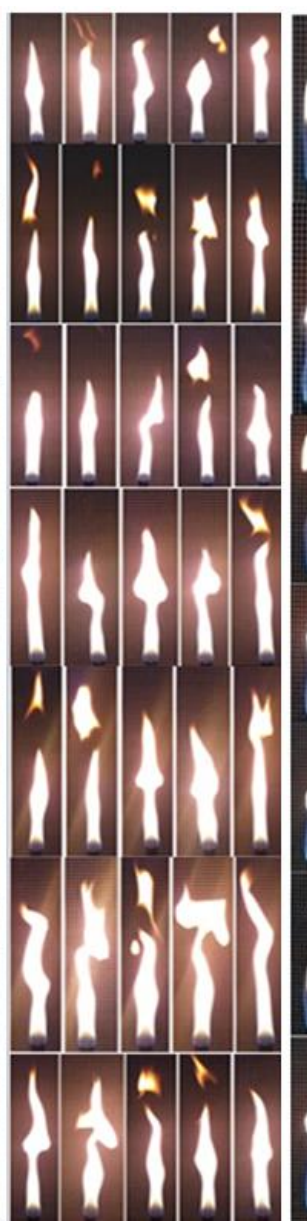

(e)

DOI: 10.5281/zenodo.1311103

Figure 9: Variation in flames with varying sound wave's frequencies for varying air entrainment configurations

Additionally, some peculiar flame structures like of a M-shape and bifurcated at the end were detected. The flame shapes were noted to be almost three-fourth blue which indicates its premixed strength. In the present case, a partially premixed flame was observed. The flickering nature was practical due to the non-linear interaction between the thermal and acoustic energy. The effects were corroborated by the wall heat interactions (heat loss), and aerodynamic flow interactions with the steady flame. Next, we look at the variation in Gross Flame Length (GFL) with impinging acoustics (fig. 11). The nearest to the base case was figured to be all holes closed case where the GFL approached the base case. Quantitatively, for all holes closed case, the GLF increased 43.34\%-61.92\% more than base case from range of 100-1000 Hz then decreased up to $\mathbf{3 5 . 3 8 \%}$ more. This is the only representative case with GFL more than the base under varying acoustics. However, the length is not fixed but varying. The values reflect small drop reaching $5000 \mathrm{~Hz}$ and again it rises to $\mathbf{5 3 . 9 6 \%}$ and with gradual drop to $\mathbf{4 3 . 3 4 \%}$ more than base case for higher acoustic frequencies of 7500 and $10000 \mathrm{~Hz}$, respectively. 


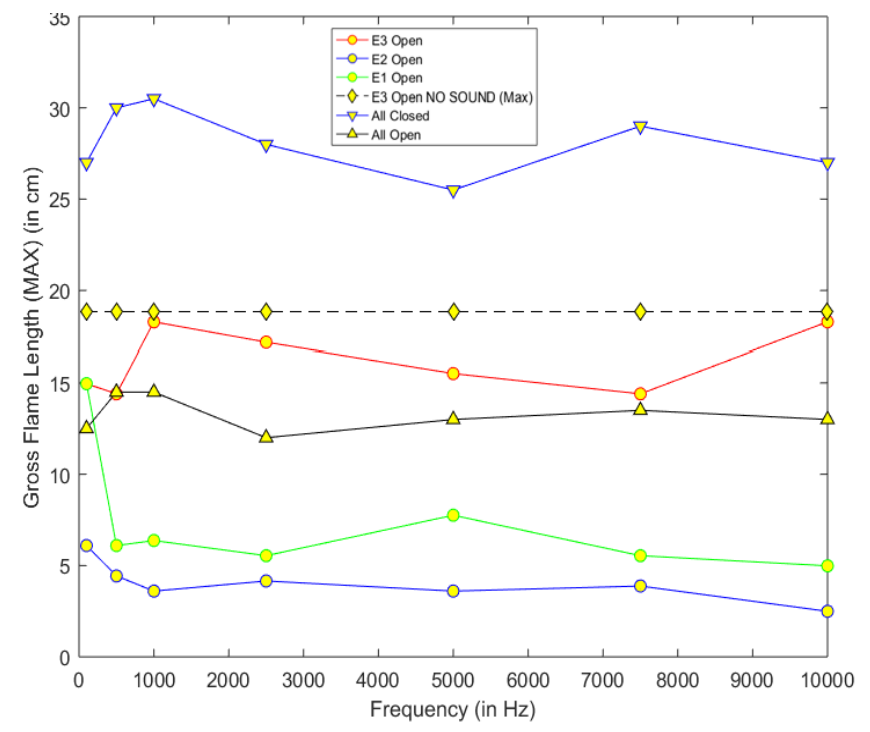

Figure 11: Varying Maximum Gross Flame Length with frequency

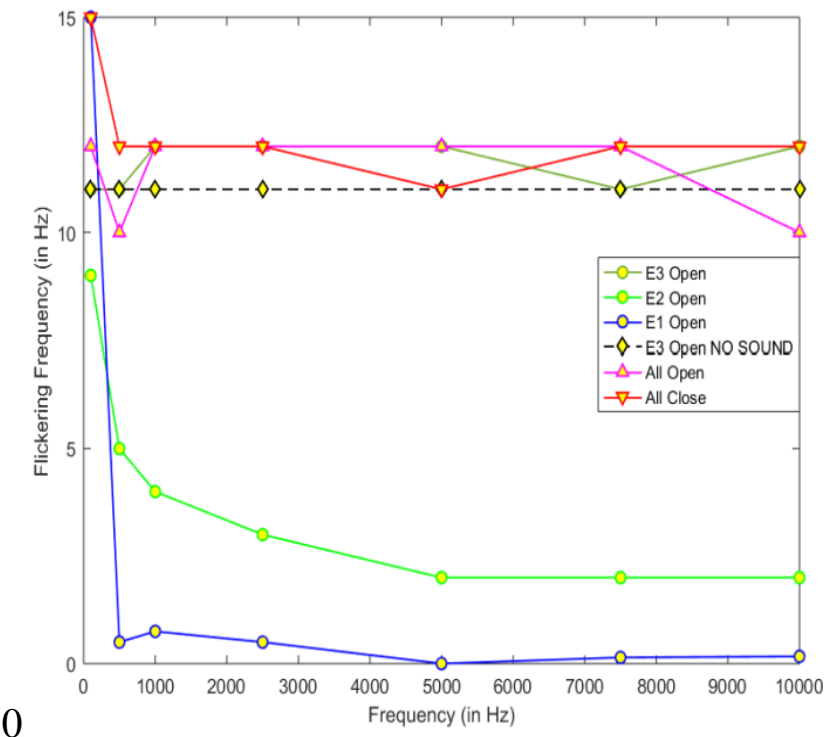

Figure 12: Variation of Flame Flickering frequency with Acoustic frequency

At $1000 \mathrm{~Hz}$ and $7500 \mathrm{~Hz}$, the local maxima of GFL reached which spreads soot portion of the flame. At 100, 5000 and $10000 \mathrm{~Hz}$, the GFL rose to gain energy by shifting the process towards complete combustion by exposing flame to more oxidizer, thus the decreasing soot area. In all rest of the cases the GFL didn't reach till base case. It is interesting to note that, E2 open and all holes open case follows the similar trends till $1000 \mathrm{~Hz}$ with the GFL decreasing and till $2500 \mathrm{~Hz}$ in all open case. Further, it continues incrementing up to $4 \%$ relatively till $7500 \mathrm{~Hz}$ and almost stays constant till $10000 \mathrm{~Hz}$. In E2 open case, after $1000 \mathrm{~Hz}$ it almost stays constant till $10000 \mathrm{~Hz}$. But in all open case and E2 open case the maximum GFL reached $\mathbf{5 7 . 3 5 \%}$ and $\mathbf{6 7 . 6 4 \%}$ less than base case. For E3 open case, 500 and $7500 \mathrm{~Hz}$ local maxima GFL decreased 23.53\% than base case. For E1 open case at $100 \mathrm{~Hz}$ in local maxima decreased up to $\mathbf{2 0 . 5 9 \%}$ than base case, $\mathbf{5 8 . 8 2 \%}$ decreased than base case at $5000 \mathrm{~Hz}$. At rest of the frequencies in E1 open case GFL maxima almost decremented $\mathbf{7 0 \%}$ than base case. 
Figure 12 shows the variation of flickering frequency with acoustics for separately E1, E2 and E3 open cases and the extreme cases of all holes closed, and all holes open cases. Looking at the plot, it was noted that, for the case of E3 open, the frequencies overshoot the base case frequency by 9 $\%$ at $1000,2500,5000$ and $10000 \mathrm{~Hz}$ acoustics. For 100, 500 and $7500 \mathrm{~Hz}$, the flame imitated the base case range of frequenting values. For only E2 open configuration, the flickering flame frequency reduced by $\mathbf{1 8 \%}$, for the highest frequency, it decreased up to $\mathbf{7 2 . 7 2 \%}$ up to $2500 \mathrm{~Hz}$ and further up to $\mathbf{8 1 . 8 2 \%}$ and stays constant as rise in acoustic frequency up to $10000 \mathrm{~Hz}$. In case of only E1 open configuration, $100 \mathrm{~Hz}$ sound wave impingement induces flame frequency $\mathbf{3 6 . 3 6 \%}$ more. A drastic drop of flickering frequency was observed up to $95.45 \%$ at $500 \mathrm{~Hz}$ and stays almost constant having error of $\mathbf{\pm 4} \%$. At $5000 \mathrm{~Hz}$, with acoustic impingement, a completely stationary flame occurred with no flame frequencies. But in case of all holes open $36.36 \%$ flickering frequency rose at $100 \mathrm{~Hz}$ and flame frequency of about $\mathbf{1 0} \%$ more and almost stays constant for all other acoustic frequencies. In all holes open configuration, the 10\% more flickering frequency was observed for almost all cases except, the 500 and $10000 \mathrm{~Hz}$ where about $\mathbf{1 0 \%}$ lesser flickering frequency was observed. As optimized here, it is very difficult to drop the flickering frequency of flame having collinear-multi-entraining holes in either fully open or fully closed cases and even for the only opened case of closest air entraining hole to the reservoir. The wavering nature owing to the multivariable effect may be attributed to the competing effect of the air-entrainment and acoustics. The overall flame structure is yielded by the resultant consequences. The energy interactions may lead to effectiveness of the central energy source with energy transferred to the system or from the system highlighting stability and thus combustion. The flickering phenomenon may be attributed to the uneven and unsteady inter-energy interactions in a flame. The soot formation/oxidation in a flame can be well optimized utilizing acoustics in a coupled arrangement with significant air-entrainment. The thermal energy in this system is contained in two forms which are namely the external and internal form, where the internal form refers to the chemical kinetics and the external thermal energy is manifested in the form of localized velocity and temperature gradient fields along the nozzle wall, the impinging acoustic energy alters the external thermal energy, which is a cause of the internal energy. Thus, with progress of time the acoustic energy alter the internal thermal energy which leads to other forms of energy interactions whose effect on the flame can be observed by observing the flame height, which is the key analysis parameter in this research work.

\section{Conclusions}

A systematic experimentation was carried out to study the effect of effect of thermo-acoustics and position of air entrainment on the soot formation. To experiment, a sound source was utilized to generate acoustics of varying frequencies, and a nozzle with sets of entrainment holes used to study the combined effect of this phenomenon on soot formation characteristics. Along with external acoustic energy, other energy interactions also affect the flame characteristics which are studied by varying the entrainment location. Based on the experimentation, following conclusions can be drawn:

1) The external acoustics doesn't always aid in the soot reduction.

2) Flame length(s) for distinct cases are utilized to yield the suitable soot reduction regimes by observing flame characteristics.

3) The energy interactions caused due to applied acoustic and thermal energy compete and the resulting effect on flame is manifested in the form of soot formation and flame height. 
4) The two energies viz., thermal and acoustic energy compete and jointly produce effects on the flame characteristics which is the soot formed.

5) The case is the most realistic analysis that matches practical scenario where the combustion-based appliance is exposed to multiple sources of external energy.

\section{References}

[1] S. H. Sohrab and C. K. Law, "Influence of Burner Rim Aerodynamics on Polyhedral Flames and Flame Stabilization". Combustion and Flame, 62:243-254.

[2] Aspi R. Wadia, "Advanced Combustor Liner Cooling Technology for Gas Turbine". Defence Science Journal, Vol38, No. 4, October 1988. pp363-380.

[3] Xiaodong Jing and Xiaofeng Sun, "Experimental investigations of perforated liners with bias flow". Journal of Acoustic Society America 106 (5), November 1999.

[4] Jeff D. Eledredge and Ann P. Dowling, "The absorption of axial acoustic waves by a perforated liner with bias flow". Journal of Fluid Mechanics (2003), vol. 485, pp. 307-335.

[5] Li Lei, Guo Zhihui, Zhang Chengyu, Sun Xiaofeng, "A Passive Method to Control Combustion Instabilities with Perforated Liner". Chinese Journal of Aeronautics 23(2010) 623-630.

[6] Claus Heuwinkel, Andre Fischer, Ingo Rohle, Lars Enghardt, and Friedrich Bake, "Characterization of a Perforated Liner by Acoustic and Optical Measurements". 16th AIAA/CEAS Aeroacoustics Conference.

[7] José M. N. Rodrigues and Edgar C. Fernandes, "Stability Analysis and Flow Characterization of Multi-Perforated Plate Premixed Burners". 17th International Symposium on Applications of Laser Techniques to Fluid Mechanics Lisbon, Portugal, 07-10 July 2014.

[8] Jiao Lei, Naian Liu, Linhe Zhang, Kohyu Satoh, "Temperature, velocity and air entrainment of fire whirl plume: A comprehensive experimental investigation". Combustion and Flame 162 (2015) 745-758.

[9] Kai Wang, Changfa Tao, Qin Liu, Yejian Qian and Peixiang He, “An experimental investigation of flame height and air entrainment rate of double jet fires". Experimental Heat Transfer: A Journal of Thermal Energy Generation, Transport, Storage, and Conversion.

[10] Longhua Hu, Shixiang Liu and Xiaolei Zhang, "Flame heights of line-source buoyant turbulent non-premixed jets with air entrainment constraint by two parallel side walls". Fuel 200 (2017) 583589.

[11] Changfa Tao, Yongqiang Liu, Fei Tanga, Qiang Wang, "An experimental investigation of the flame height and air entrainment of ring pool fire". Fuel 216 (2018) 734-737.

[12] Deepika Ram, Vikram Ramanan, Vinayak Malhotra and Hrishikesh Srivatsav, "Soot Suppression in Laminar Jet Diffusion Flames by Shear". AIAA SciTech Forum, 2018 AIAA Aerospace Sciences Meeting, 8-12 January 2018, Kissimmee, Florida, DOI: 10.2514/6.2018-1413.

\footnotetext{
*Corresponding author.

E-mail address: vinn99@ gmail.com
} 